\title{
Evaluation of systemic and mucosal immune responses in mice administered with recombinant Salmonella Typhimurium expressing IutA protein
}

\author{
In-Gyeong Oh, Minsu Choi, John Hwa Lee* \\ College of Veterinary Medicine and Bio-Safety Research Institute, Chonbuk National University, Jeonju 561-756, Korea \\ (Received: January 4, 2013; Revised: May 3, 2013; Accepted: July 16, 2013)
}

\begin{abstract}
Avian pathogenic Escherichia coli (APEC) are known to cause extraintestinal disease in poultry, leading to substantial losses in the industry. IutA, iron-regulated aerobactin receptor is firmly associated with APEC. To assess the potential of IutA to induce protective immune responses, attenuated Salmonella Typhimurium strain expressing IutA was constructed and administered orally to BALB/c mice. The IutA-specific immune responses were measured with sera, vaginal and fecal samples by an enzyme-linked immunosorbent assay. We found that the Salmonella-IutA vaccine induced significantly higher immune responses as compared to the control inoculated with the attenuated $S$. Typhimurium containing the plasmid only. The IutA-specific immune responses were increased by second immunization at third week after initial immunization, whereas triple immunization induced lower immune responses than those induced by the double immunization. The Salmonella-IutA vaccine induced a nature of immunity biased to the Th1type, as judged by the ratio of IutA-specific IgG isotypes (IgG2a/IgG1). Overall, these results suggest that the Salmonella-IutA vaccine appear to be suitable candidate for a vaccine against APEC.
\end{abstract}

Keywords : avian pathogenic Escherichia coli, boost immunization, IutA, oral vaccination

\section{Introduction}

Escherichia (E.) coli is a normal inhabitant of the intestinal tract of humans and animals. However, avian pathogenic E. coli (APEC) is a frequent cause of extraintestinal infections, collectively called colibacillosis, in poultry. In the past few years, both the incidence and severity of colibacillosis have increased rapidly, and current trends indicate that it is likely to continue and become an even greater problem in the poultry industry $[1,2,4]$. The control efforts on colibacillosis have been hampered by limited understanding of the mechanisms of disease caused by APEC. Management approaches designed to reduce predisposing conditions and prevention using antibacterial agents may not be as effective in the future, due to significant and continuous increases in the frequencies of resistances to antibiotics [9]. A valuable alternative for APEC infection is the prevention by vaccination. Oral vaccination has specific advantages in terms of convenience of administration, induction of effective mucosal immune responses as well as systemic immune responses [22]. Because APEC principally infects respiratory tract of poultry, induction of secretory IgA responses by oral vaccination is potentially effective strategies against APEC.

The IutA gene encode the iron-regulated aerobactin receptor which compete with transferrins to scavenge iron in the host environment [3]. Previous reports showed that IutA is an essential iron receptor gene in approximately $90 \%$ of APEC strains $[12,21]$ and the IutA protein has been considered a possible candidate for a vaccine against $E$. coli respiratory and septicemic disease in chickens [8], since anti-IutA antibodies were protective against heterologous challenge as well as homologous challenge.

Attenuated Salmonella strains have been developed as carriers for the delivery of heterologous antigens to the mammalian immune systems [6]. Oral immunization with recombinant Salmonella vaccine expressing pneumococcal surface protein $\mathrm{A}$ induced effective protection against pnemococci infection in mice [7]. The objective of the present study was: 1) construction of recombinant attenuated Salmonella vaccine strain expressing IutA antigen, 2) evaluation whether immunoprotective responses were induced by oral immunization with Salmonella-IutA vaccine, and 3) assessment of the need for boost immunization.

\section{Materials and Methods}

\section{Bacterial strains, animals, growth conditions}

Inbred 6-week-old female BALB/c mice (Koatech, Korea) were used for immunization and were maintained at animal facility of Chonbuk National University under standard con-

*Corresponding author

Tel: +82-63-270-2553, Fax: +82-63-270-3780

E-mail: johnhlee@chonbuk.ac.kr 
ditions according to the Institutional Guidelines. The 4 crp-28 $\triangle a s d A 16$ attenuated Salmonella $(S$.) Typhimurium, $\chi 8501$ and the $\mathrm{Asd}^{+}$plasmids, pYA3493 and pYA3560 were kindly provided by Ho Young Kang, Department of Microbiology, College of Natural Sciences, Pusan National University, Korea. The IutA ${ }^{+}$E. coli strain, JOL718 was used as a source of genomic DNA. The bacterial strains were grown $37^{\circ} \mathrm{C}$ in either LB (Difco, USA) broth or agar. When required, antibiotics were added to the culture media. Diaminopimelic acid (DAP) (Sigma-Aldrich, USA) was added for the growth of the Asd $^{-}$strains.

\section{Purification of IutA protein}

The gene fragment of iutA was obtained by polymerase chain reaction using a pair of primers, (F,5'CGTGGATCCG CAACAAACCGATGATGAAACG3';R,5'CGGAAGCTTT CAGAACAGCACAGAGTAGTTCAGACC3') and were cloned into the pQE10 (Qiagen, Germany). This recombinant plasmids were transformed into a $E$. coli M15 (Qiagen). In-frame cloning was confirmed by nucleotide sequencing. The purified protein was obtained by an affinity purification process with $\mathrm{Ni}^{2+}$-nitrilotriacetic acid-agarose support (Qiagen). The protein was verified by sodium dodecyl sulfate-polyacrylamide gel electrophoresis (SDS-PAGE), and protein concentration was estimated using a protein assay system (Bio Rad, USA).

\section{Production of antibodies to IutA protein}

New Zealand White rabbits were used in the production of antibodies. A primary dose of $250 \mu \mathrm{g}$ of purified IutA protein in Freud's complete adjuvant (Sigma-Aldrich) was administered subcutaneously to rabbits followed by two additional doses of protein $(250 \mu \mathrm{g})$ emulsified in Freud's incomplete adjuvant at 2 weeks intervals. The rabbits were bled at 2nd week after the last administration of dose and the sera were collected.

\section{Preparation of the Salmonella-IutA vaccine strain}

The gene of IutA was cloned into pYA3560. The attenuated $S$. Typhimurium, $\chi 8501$ were transformed with this cloned plasmid by electroporation and then plated on LB plates without DAP. Only clones harboring the recombinant plasmid were cultured under these conditions. The expression of the antigen was assessed by Western blotting.

\section{Western blotting}

The bacterial cultures were centrifuged at $7,000 \times \mathrm{g}$ for 10 min, and the supernatant were collected for analysis of secreted antigens. The supernatant was concentrated for $1 \mathrm{~h}$ in ice-cold $10 \%$ trichloroacetic acid solution. For Western blotting, proteins separated via SDS-PAGE were transferred to $0.2 \mu \mathrm{m}$ microporous polyvinylidene fluoride membranes (EMD Millipore, USA), and then incubated at $4^{\circ} \mathrm{C}$ overnight with rabbit polyclonal antisera against IutA. Membranes were then incubated with a goat anti-rabbit IgG conjugated to horseradish peroxidase (Pierce, USA) and reactive protein were visualized using a immunoblot detection system (Intron, Korea).

\section{Immunization and collection of samples}

Groups of five mice were immunized three times at 3week intervals with $2 \times 10^{9}$ colony-forming units of Salmonella-IutA vaccine by the oral route. Prior to orally immunization, food and water were withdrawn $4 \mathrm{~h}$. The mice treated with the attenuated Salmonella containing pYA3493 only served as the control. Blood was obtained by retro-orbital puncture with heparinized capillary tubes and vaginal samples were obtained by $100 \mu \mathrm{L}$ PBS wash. The fecal samples were weighed and resuspended in PBS containing $0.1 \%$ sodium azide [20]. Samples were collected biweekly intervals and stored at $-20^{\circ} \mathrm{C}$ until used.

\section{Measurement of immune responses by ELISA}

For enzyme-linked immunosorbent assays (ELISA), Polystyrene 96-well flat-bottom microtiter plates were coated with IutA protein and incubated overnight at $4^{\circ} \mathrm{C}$. To block nonspecific protein binding, the plates were incubated for $1 \mathrm{~h}$ at $37^{\circ} \mathrm{C}$ with $\mathrm{PBS}$ containing $3 \%$ skim milk. Vaginal fecal and serum samples were diluted $1: 4,1: 4$ and $1: 100$, respectively and then diluted samples were incubated for $1.5 \sim 2 \mathrm{~h}$ at $37^{\circ} \mathrm{C}$. Anti-mouse immunoglobulin-horseradish peroxidase conjugate (Southern Biotechnology, USA) was used as the second antibody and the reaction was visualized with $o$-phenylenediamine (Sigma-Aldrich). Finally, optical densities at $492 \mathrm{~nm}$ were determined using an automated ELISA spectrophotometer (TECAN, Austria).

\section{Statistical analysis.}

Independent-Samples $t$-test was used to determine significant differences in antibody titers between double dose immunized and single dose immunized groups, and significant differences in the antibody titers of IgG2a and IgG1 in immunized groups. Statistical results were considered significant when $p$ values were $<0.05$. All statistical analyses were carried out with the SPSS 16.0.1 program (IBM SPSS statistics; IBM, USA).

\section{Results}

\section{Construction of a $S$. Typhimurium vaccine expressing IutA protein}

The $S$. Typhimurium strain, $\chi 8501$ transformed with recombinant plasmid stably expressed IutA as detected by development of Western immunoblots (Fig. 1). The IutA was detected in the concentrated culture supernatant of Salmonella-IutA vaccine, but was not detected in that of control.

Anti-IutA immune responses in mice following oral immunization with Salmonella-IutA vaccine

The systemic IgG responses in sera and mucosal IgA responses in vaginal and fecal samples of mice were mea- 


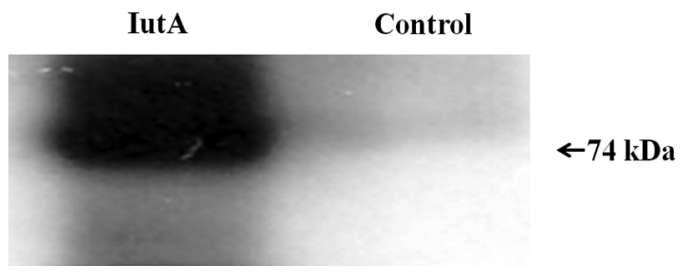

Fig. 1. Western immunoblot analyses of $S$. Typhimurium strain expressing IutA antigen. The secreted proteins in culture supernatant were subjected to sodium dodecyl sulfate-polyacrylamide gel electrophoresis, transferred to PVDF membrane and probed with the rabbit anti-IutA serum. Right: Salmonella-IutA vaccine, Left: the control. Sizes $(74 \mathrm{kDa})$ of the proteins are shown at the right.

(A)

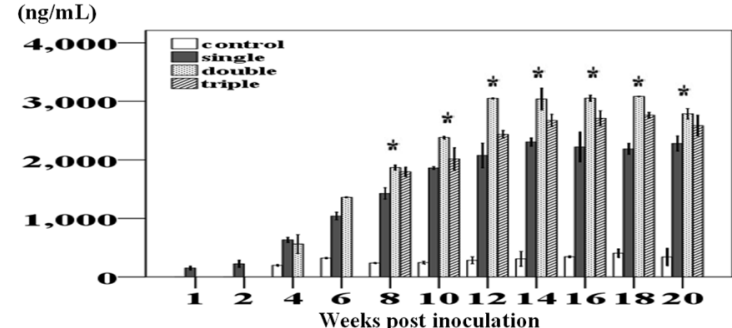

(B)

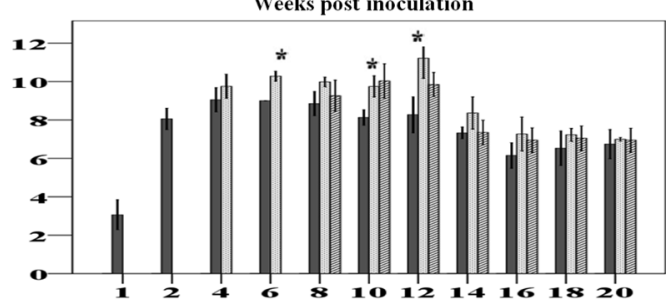

(C)

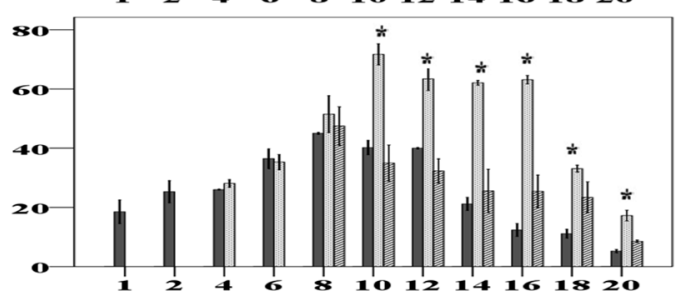

Fig. 2. Anti-IutA immune responses in mice orally immunized with Salmonella-IutA vaccine strain at 3-week intervals. (A) Anti-IutA IgG titers in sera. (B) Anti-IutA IgA titers in vaginal samples. (C) Anti-IutA IgA titers in fecal samples. Immunization groups of single dose immunization (gray), double dose immunization (dot), triple dose immunization (comb) and the control (white) were shown as indicated. Asterisks indicate a significant difference between the values of double dose immunized mice $\left({ }^{*} p<0.05\right)$, and those of single dose immunized mice.

sured. The Salmonella-IutA vaccine induced both systemic and mucosal immune responses in mice. Serum IutA-specific $\mathrm{IgG}$ responses in immunized groups with the vaccine were much higher than those in the control group. The double immunization induced the highest anti-IutA IgG titers, followed by the triple immunization and the single immunization. The levels of IgG antibody to IutA were highest at 14th week, this remained until the end of the study (Fig. 2A). In vaginal samples, anti-IutA IgA titers in the double immu-

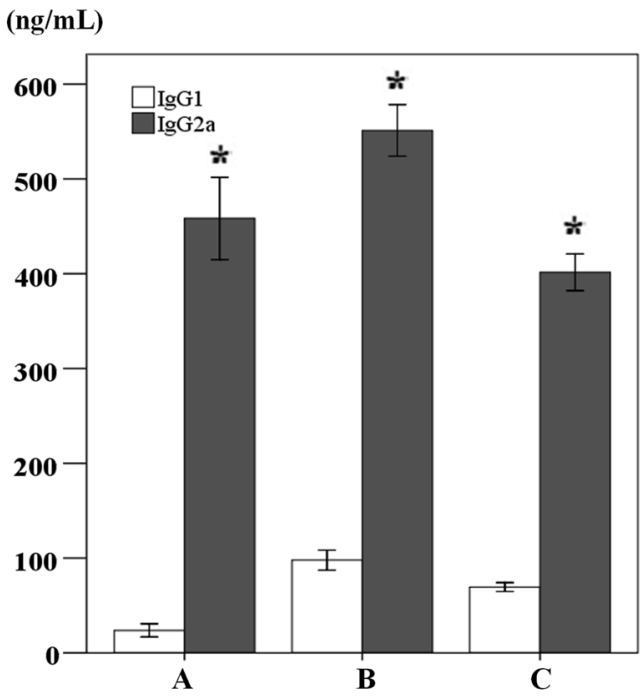

Fig. 3. Anti-IutA isotypes produced in response to the Salmonella-IutA vaccine strain. The $\mathrm{X}$ axis shows the dose of vaccine administered. (A) single dose immunization. (B) double dose immunization. (C) triple dose immunization. Asterisks indicate a significant difference between the antibody titers of $\operatorname{IgG} 2 \mathrm{a}$ $\left({ }^{*} p<0.05\right)$, and IgG1 in immunized groups.

nization group were generally higher than those in the single and triple immunization group, and they also showed a slight decline over the 20 weeks period of the study. The IgA responses in the control group were virtually undetectable in the vaginal and fecal samples (Fig. 2B). In fecal samples, anti-IutA $\operatorname{IgA}$ responses in double immunization group were increased significantly than those in the single immunization group. The anti-IutA IgA levels in the single and double immunization groups continued to decline from the 12th week and the 16th week, respectively. The anti-IutA IgA titers in the triple immunization group were lower than those in the single immunization group (Fig. 2C). When the distribution of IutA specific IgG isotypes at 12 th week was analyzed, mice immunized with Salmonella-IutA vaccine induced high levels of $\operatorname{IgG} 2 \mathrm{a}$ antibody and low levels of IgG1 antibodies (Fig. 3).

\section{Discussion}

Control of avian colibacillosis using vaccines may be a most effective regimen, thus there have been many attempts to develop vaccines and have been tested experimentally [10, 13, 16]. Many of these were effective against homologous APEC challenge but not against heterologous challenge. Thus, new vaccine strategies were required to protect APEC which are quite diverse in terms of serogroup [15]. One of the virulence factors, IutA is present at high frequency in APEC of various serogroups [11, 12]. Kariyawasam et al. [8] also reported that anti-IutA antibodies were effective against heterologous challenge. Thus, it is thought that IutA-based 
vaccine may induce protection against a wide range of APEC.

Attenuated Salmonella strains have been developed as carriers for the delivery of heterologous antigens to the mammalian immune systems. In this study, the recombinant attenuated Salmonella vaccine for IutA virulence factor was constructed using $S$. Typhimurium as a live vehicle for oral delivery of IutA, and secretion of IutA from this vaccine strain was confirmed by the immunoblot assay. It was demonstrated that $S$. Typhimurium producing IutA was able to elicit significant anti-IutA systemic and mucosal immune responses via oral administration to mice. Given that the major infection route of APEC is respiratory mucosa, the mucosal IgA immune response would be essential to prevent infection of APEC. This induction of mucosal immune responses is one of the several benefits of immunization by oral route.

Double immunization induced higher immune responses than those induced by single dose of immunization. Notably, enhanced mucosal IgA responses of fecal samples in double immunization were maintained high for long period. Ramasamy et al. [14] reported that single oral immunization failed to induce the satisfactory antigen-specific antibodies and also found that frequency in oral vaccination schedule would be required for the induction and the maintenance of mucoal IgA levels. Interestingly, triple immunization induced lower immune responses than those induced by double immunization. It seemed that triple immunization could induce immune tolerance but the mechanisms by which mucosal tolerance is mediated are not clearly understood.

Induction of the appropriate type of antigen-specific immune responses is critical for the success of vaccines. The induction of Th1 type responses is preferential for vaccines against intracellular pathogens [5, 19]. T-helper type1 (Th1) cells induce $\mathrm{B}$ cells to produce the complement-activating antibody $\operatorname{IgG} 2 \mathrm{a}$, while non complement-activating IgG1 is the predominant isotype induced in a Th2 cells [17, 18]. In this study, the distribution of IutA-specific IgG isotypes (IgG2a and $\operatorname{IgG} 1$ ) was analyzed to evaluate the nature of immunity. The animals were immunized with Salmonella-IutA vaccine produced high levels of IgG2a and low levels of IgG1 antibodies. This result suggested that this vaccine induced Th1 biased responses, which is suitable for protection of intracellular pathogens such as APEC and it might contribute in future studies to optimize vaccine regimen against infections of APEC highly frequent in poultry industry.

\section{Acknowledgments}

This work was supported by Mid-career Researcher Program through National Research Foundation (NRF) grant funded by the Ministry of Education, Science and Technology (MEST) (No. 2012-R1A2A4A01002318), Korea.

\section{References}

1. Altekruse SF, Elvinger F, Lee KY, Tollefson LK, Pierson
EW, Eifert J, Sriranganathan N. Antimicrobial susceptibilities of Escherichia coli strains from a turkey operation. J Am Vet Med Assoc 2002, 221, 411-416.

2. Delicato ER, de Brito BG, Gaziri LCJ, Vidotto MC. Virulence-associated genes in Escherichia coli isolates from poultry with colibacillosis. Vet Microbiol 2003, 94, 97-103.

3. de Lorenzo V, Bindereif A, Paw BH, Neilands JB. Aerobactin biosynthesis and transport genes of plasmid ColV-K30 in Escherichia coli K-12. J Bacteriol 1986, 165, 570-578.

4. Ewers C, Janßen T, Kießling S, Philipp HC, Wieler LH. Molecular epidemiology of avian pathogenic Escherichia coli (APEC) isolated from colisepticemia in poultry. Vet Microbiol 2004, 104, 91-101.

5. Graham MB, Braciale VL, Braciale TJ. Influenza virusspecific $\mathrm{CD}^{+} \mathrm{T}$ helper type $2 \mathrm{~T}$ lymphocytes do not promote recovery from experimental virus infection. J Exp Med 1994, 180, 1273-1282.

6. Gulig PA, Curtiss R 3rd. Plasmid-associated virulence of Salmonella typhimurium. Infect Immun 1987, 55, 2891-2901.

7. Kang HY, Srinivasan J, Curtiss R 3rd. Immune responses to recombinant pneumococcal PspA antigen delivered by live attenuated Salmonella enterica serovar Typhimurium vaccine. Infect Immun 2002, 70, 1739-1749.

8. Kariywasam S, Wilkie BN, Gyles CL. Resistance of broiler chickens to Escherichia coli respiratory tract infection induced by passively transferred egg-yolk antibodies. Vet Microbiol 2004, 98, 273-284.

9. Kim TE, Jeong YW, Cho SH, Kim SJ, Kwon HJ. Chronological study of antibiotic resistances and their relevant genes in Korean avian pathogenic Escherichia coli isolates. J Clin Micorbiol 2007, 45, 3309-3315.

10. Kwaga JKP, Allan BJ, van den Hurk JV, Seida H, Potter AA. A car $A B$ mutant of avian pathogenic Escherichia coli serogroup $\mathrm{O} 2$ is attenuated and effective as a live oral vaccine against colibacillosis in Turkeys. Infect Immun 1994, 62, 3766-3772.

11. Lafont JP, Dho M, D'Hauteville HM, Bree A, Sansonetti PJ. Presence and expression of aerobactin genes in virulent avian strains of Escherichia coli. Infect Immun 1987, 55, 193-197.

12. Ons E, Bleyen N, Tuntufye HN, Vandemaele F, Goddeeris BM. High prevalence iron receptor genes of avian pathogenic Escherichia coli. Avian Pathol 2007, 36, 411414.

13. Peighambari SM, Hunter DB, Shewen PE, Gyles CL. Safety, immunogenicity, and efficacy of two Escherichia coli cya crp mutants as vaccines for broilers. Avian Dis 2002, 46, 287-297.

14. Ramasamy R, Yasawardena S, Zomer A, Venema G, Kok J, Leenhouts K. Immunogenicity of a malaria parasite antigen displayed by Lactococcus lactis in oral immunisations. Vaccine 2006, 24, 3900-3908.

15. Rodriguez-Siek KE, Giddings CW, Doetkott C, Johnson TJ, Nolan LK. Characterizing the APEC pathotype. Vet Res 2005, 36, 241-256.

16. Roland K, Karaca K, Sizemore D. Expression of Escherichia coli antigens in Salmonella typhimurium as a vaccine to prevent airsacculitis in chickens. Avian Dis 2004, 48, 595-605. 
17. Stevens TL, Bossie A, Sanders VM, Fernandez-Botran R, Coffman RL, Mosmann TR, Vitetta ES. Regulation of antibody isotype secretion by subsets of antigen-specific helper T cells. Nature 1988, 334, 225-258.

18. Strindelius L, Filler M, Sjöholm I. Mucosal immunization with purified flagellin from Salmonella induces systemic and mucosal immune responses in $\mathrm{C} 3 \mathrm{H} / \mathrm{HeJ}$ mice. Vaccine 2004, 22, 3797-3808.

19. Yamamura M, Uyemura K, Deans RJ, Weinberg K, Rea TH, Bloom BR, Modlin RL. Defining protective responses to pathogens: cytokine profiles in leprosy lesions. Science 1991, 254, 277-279.

20. Yoon HA, Aleyas AG, George JA, Park SO, Han YW, Hyun BH, Lee JH, Song HJ, Cho JG, Eo SK. Correlation between the nature of immunity induced by different immunogens and the establishment of latent infection by wild-type pseudorabies virus. Res Vet Sci 2007, 83, 73-81.

21. Vandekerchove D, Vandemaele F, Adriaensen C, Zaleska M, Hernalsteens JP, De Baets L, Butaye P, Van Immerseel F, Wattiau P, Laevens $H$, Mast J, Goddeeris B, Pasmans F. Virulence-associated traits in avian Escherichia coli: comparison between isolates from colibacillosisaffected and clinically healthy layer flocks. Vet Microbiol 2005, 108, 75-87.

22. Zhao X, Zhang M, Li Z, Frankel FR. Vaginal protection and immunity after oral immunization of mice with a novel vaccine strain of Listeria monocytogenes expressing human immunodeficiency virus type 1 gag. J Virol 2006, 80, 8880-8890. 